\section{OP52 THE PREVALENCE AND CORRELATES OF OBJECTIVELY MEASURED SEDENTARY TIME IN PREGNANT WOMEN AT RISK OF GESTATIONAL DIABETES IN THE UK: A MIXED METHODS STUDY}

JM Wagnild*, HL Ball, TM Pollard. Anthropology, Durham University, Durham, UK

\subsection{6/jech-2018-SSMabstracts.52}

Background Despite increasing interest in the impact of sedentary behaviour on health, little is known about sedentary behaviour during pregnancy. Given the association between sedentary behaviour and risk of type 2 diabetes, it is particularly important to understand the prevalence and correlates of sedentary behaviour during pregnancy in women at risk of gestational diabetes. The aims of this mixed methods study were to 1) quantify objectively measured sedentary time during pregnancy in women at risk of gestational diabetes, 2) quantitatively examine the correlates of objectively measured sedentary time, and 3) explore the place of sedentary behavior and physical activity in these pregnant women's lives using qualitative data.

Methods Pregnant women $(n=192)$ recruited from two hospitals in the North East of England who had a risk factor for gestational diabetes continuously wore activPAL accelerometers for seven days during the second trimester, and a subsample of participants $(n=18)$ took part in a semi-structured interview in the third trimester. Multiple linear regression analyses were applied to accelerometry data and thematic analysis was conducted with interview data using NVivo11.

Results On average, women spent 9.57 hours per day $(\mathrm{SD}=1.62)$ engaged in sedentary behaviour, which accounted for $71.7 \%$ of waking hours. In multivariate linear regression models, the only statistically significant predictor of sedentary time was Index of Multiple Deprivation. Time spent sedentary was 9.29 hours (95\% CI 9.00 to 9.57) for the most deprived tertile, 10.26 hours (95\% CI 9.74 to 10.78 ) for the middle tertile, and 9.81 hours (95\% CI 9.29 to 10.33) for the least deprived tertile. The key overarching theme that emerged from the interview data was that there was a social expectation that the participants should slow down and sit down simply due to their pregnancy status, which often conflicted with participants' own perspectives that their roles in everyday life were incompatible with 'sitting around.'

Conclusion These findings indicate that sedentary time during pregnancy may be socially patterned such that those residing in the most deprived areas spend significantly less time sedentary than those in less deprived areas. Furthermore, the reported strong social expectation for pregnant women to slow down and halt their everyday lives is a challenge for interventions to reduce sedentary time and increase physical activity during pregnancy.

\section{OP53 THE ASSOCIATION BETWEEN MODE OF DELIVERY AT BIRTH AND WHEEZING TRAJECTORIES - EVIDENCE FROM THE UK MILLENNIUM COHORT STUDY}

N Alterman*, JJ Kurinczuk, MA Quigley. National Perinatal Epidemiology Unit, University of Oxford, Oxford, UK

\subsection{6/jech-2018-SSMabstracts.53}

Background Delivery by caesarean section has been identified as a risk factor for asthma. Meta-analyses have reported a $20 \%$ increased risk for childhood asthma or its common symptom - wheezing. However, asthma is a heterogeneous disease with distinct phenotypes portrayed as various wheezing trajectories. We sought to assess the relationship between mode of delivery at birth and three commonly identified wheezing trajectories of childhood, as well as additional asthma-related outcomes.

Methods Data were drawn from the UK Millennium Cohort Study and included 8744 participants who were singleton, born at term and healthy at the time of birth. Participating families were interviewed at infancy and at ages 3, 5, 7 and 11 years. Wheezing trajectories were examined through parental report of wheezing in the past year, which was coded as 'transient' (resolved by age 5) 'persistent' (both before and after age 5) or 'late-onset' (appearing starting age 5) compared to no report of wheezing. Relative risk ratios were estimated using multinomial logistic regression. Cross-sectional asthmarelated outcomes of recent wheezing, severe recent wheezing, ever having asthma and asthma medication use were estimated using logistic regression.

Results Most of the children were born through unassisted vaginal delivery (72\%); instrumental vaginal delivery, planned caesarean and emergency caesarean accounted for 10\%, 9\% and $10 \%$ of births respectively. The proportion of children exhibiting transient, persistent and late-onset wheezing was $11.6 \%, 11.1 \%$ and $12.9 \%$ respectively.

Children born by a medically interventional delivery had similar odds of experiencing transient wheezing compared to no wheezing as those born by unassisted vaginal delivery. The same was true for late-onset wheezing compared to none. The risk of experiencing persistent wheezing was higher in children born by planned caesarean compared to those born by unassisted vaginal delivery $(\mathrm{RRR}=1.33,95 \% \mathrm{CI} \quad(1.02,1.75))$. Confounders accounted for were mother's age, education, marital status, socioeconomic position, smoking and parents' asthma in addition to child's sex, ethnicity, gestational age, birthweight and being firstborn. After adjustment, the elevated risk for persistent wheezing in the planned caesarean group remained unchanged and marginally significant $(R R R=1.32$ 95\% CI $(1.00,1.75))$.

No consistent pattern of association between mode of delivery and additional asthma-related cross-sectional outcomes was found.

Conclusion Birth by planned caesarean section may be a risk factor for persistent wheezing in childhood, but not for the transient or late-onset trajectories of wheezing. However, the strength of evidence is low due to multiple comparisons and the absence of a consistent trend of associations with additional asthma-related outcomes.

\section{OP54 ALCOHOL CONSUMPTION IN PREGNANCY AND CHILDHOOD HEARING AND NEURODEVELOPMENTAL PROBLEMS IN THE UK: ANALYSIS FROM THE MILLENNIUM COHORT STUDY}

${ }^{1}$ VS Straatmann, ${ }^{1}$ DC Taylor-Robinson, ${ }^{2}$ EH Bergh, ${ }^{1} \mathrm{KM}$ Fleming ${ }^{*}$. 'Department of Public Health and Policy, University of Liverpool, Liverpool, UK; ${ }^{2}$ Department of Audiology, Alder Hey Children's NHS Foundation Trust, Liverpool, UK

\subsection{6/jech-2018-SSMabstracts.54}

Background Fetal Alcohol Spectrum Disorders (FASD) is the leading preventable cause of disability in children in the UK. Identification and diagnosis of FASD is poor, with many children receiving diagnoses of other neurodevelopmental deficit 
(including Attention Deficit Hyperactivity Disorder -ADHD), but no FASD diagnosis. FASD is associated with a large number of comorbid conditions, including an 80-fold increased risk of sensorineural hearing loss. Using a contemporary UK birth cohort we examined the association between maternal alcohol consumption in pregnancy, hearing problems at age 3 and later ADHD.

Methods Analysis of the UK Millennium Cohort Study (MCS), based on 9179 children participating in 3 survey sweeps (9 months, 3, 7 years old). The main outcome was parentalreported hearing problems, at age 3 . We also examined selfreported diagnosis of $\mathrm{ADHD}$ at age 7 . Incident rate ratios (IRRs; 95\% confidence intervals [CI]) were estimated using Poisson regression, according to maternal drinking in pregnancy in units per week (categorised as none, 1-7, 8-14 and 15 or more) adjusted for measures of childhood socioeconomic conditions. Analyses were conducted using Stata/SE with svy commands to account for the sampling design and attrition.

Results At age 3, 1328 children (14.4\%) had reported a hearing problem with the most common of these being otitis media and unspecified hearing loss. Children of mothers who reported drinking 15 or more units per week of alcohol in pregnancy were twice as likely to have hearing problems compared to mothers who reported never drinking in pregnancy (aIRR1.9(95\% CI[1.07, 3.39])). Risk of ADHD diagnosis at age 7 was also elevated in children of mothers who drank 15 or more units per week though this was not statistically significant at the 5\% level (aIRR2.79(95\% CI[0.91, 8.57])). Children with hearing problems at age 3 were 3.5 times more likely to have a diagnosis of ADHD at age 7 (aIRR3.49 (95\% CI[1.92, 6.32])).

Conclusion In a representative UK child cohort we found a significant prevalence of parental reported hearing problems at age 3 which was associated with high maternal alcohol consumption in pregnancy. Hearing problems were associated with a higher risk of ADHD. Self-reported data of both alcohol consumption and hearing problems is a limitation of this study. Maternal alcohol consumption is rarely captured in child health records in the UK making follow-up of potentially exposed children difficult. Further investigation in children with hearing loss could lead to earlier diagnosis of neurodevelopmental conditions, including FASD, and provide opportunities to intervene and improve outcomes.

\section{OP55 THE IMPACT OF NEWBORN SCREENING ON OUTCOMES AND INEQUALITIES IN CYSTIC FIBROSIS: A UK REGISTRY BASED STUDY}

${ }^{1}$ DK Schlüter*, ${ }^{2} \mathrm{C}$ Dryden, ${ }^{3} \mathrm{KW}$ Southern, ${ }^{1} \mathrm{PJ}$ Diggle, ${ }^{4} \mathrm{D}$ Taylor-Robinson. ${ }^{1} \mathrm{CHICAS}$, Lancaster Medical School, Lancaster University, Lancaster, UK; ${ }^{2}$ Department of Paediatrics, University Hospital Wishaw, Wishaw, UK; ${ }^{3}$ Department of Women's and Children's Health, University of Liverpool, Liverpool, UK; ${ }^{4}$ Department of Public Health and Policy, University of Liverpool, Liverpool, UK

10.1136/jech-2018-SSMabstracts.55

Background Cystic fibrosis (CF) is the commonest inherited disease in white populations, and as a classically inherited genetic disease, there is no socio-economic gradient in incidence. Despite this, we have previously demonstrated early inequalities in CF outcomes such as reduced growth and poorer lung function in children from more socio-economically disadvantaged areas. In the present study, we used data from the UK CF Registry to examine the impact of newborn bloodspot screening (NBS), introduced in 2007, on inequalities in clinical outcomes in children with CF born in the new millennium.

Methods We carried out longitudinal analyses of data on 4117 individuals with CF born between 2000 and 2014 who are captured in the UK CF Registry. Clinical outcomes were the trajectories of lung function measured using percent predicted $\mathrm{FEV}_{1}\left(\mathrm{ppFEV}_{1}\right)$ from age five, weight and body mass index (BMI) z-scores from age one, and time to chronic Pseudomonas Aeruginosa (PA) infection. We developed longitudinal models for $\mathrm{ppFEV}_{1}$, weight, and BMI and a time-to-event model for PA infection to assess the association of NBS with outcomes and potential interactions with childhood socio-economic conditions (SECs), measured by the index of multiple deprivation, whilst adjusting for sex, genotype, birth cohort, ethnicity, and pancreatic insufficiency.

Results Complete data for the analyses of the effect on lung function, weight, BMI and time to chronic PA infection were available for 2267, 3424, 3410 and 3428 individuals, respectively. About one third of the individuals were diagnosed by NBS. NBS was associated with a shallower rate of lung function decline $(0.45 ; 95 \%$ CI 0.13 to 0.76 per year), and higher average weight trajectory intercept $(0.14$; $95 \%$ CI 0.06 to 0.23 standard deviations) as well as increased time to chronic PA infection. We found no significant association of NBS with the intercept for lung function or BMI; or with longitudinal trajectories of weight and BMI. There was no significant interaction between NBS and childhood SECs.

Conclusion Analyses of data from a large national CF Registry show that NBS is associated with better lung function and increased weight for all children with $\mathrm{CF}$, but there is no evidence that it has narrowed health inequalities.

\section{Smoking and youth}

\section{OP56 ABSTRACT WITHDRAWN}

\section{OP57 \#DOES PARENTAL SMOKING EXPLAIN IMPACTS OF SMOKE-FREE PUBLIC PLACES LEGISLATION ON YOUTH SMOKING INITIATION IN THE UK?}

PE Anyanwu*, P Craig, SV Katikireddi, MJ Green. MRC/CSO Social and Public Health Sciences Unit, University of Glasgow, Glasgow, UK

\subsection{6/jech-2018-SSMabstracts.56}

Background Evidence on the impact of the smoke-free public places legislation in the UK on youth smoking initiation is not well established. Changes in parental smoking behaviour may be a major mechanism by which smoke-free legislation impacts on youth smoking. Smoke-free legislation could also displace parental smoking behaviour into the home (by restricting alternative smoking spaces) or out of the home (by increasing awareness of risks to others from second-hand smoke exposure), potentially either strengthening or weakening its influence. We investigated how much of any impact of the legislation on youth smoking initiation could be explained by parental smoking, and whether associations between parental smoking and youth smoking initiation differed before or after the legislation. 